\title{
Morbus Dowling-Degos in genitoperianaler Lokalisation bei Mutter und Tochter
}

\author{
R. Jafari \\ M. Tronnier \\ F. Vakilzadeh
}

\author{
Morbus Dowling-Degos in Genitoperianal Localisation \\ in a Mother and Daughter
}

\section{Zusammenfassung}

Eine 31-jährige Frau sowie ihre 50-jährige Mutter zeigten retikuläre Hyperpigmentierungen genital und perianal. Die Diagnose eines Morbus Dowling-Degos in ausschließlich genitoperianaler Lokalisation wurde anhand histologischer und immunhistochemischer Untersuchungen gestellt.

\section{Abstract}

A 31-year-old woman and her 50-year-old mother presented genital and perianal reticular pigmented lesions. The diagnosis of Dowling-Degos disease has been established by clinical and histopathologic findings.

\section{Einleitung}

Beim Morbus Dowling-Degos (,reticulate pigmented anormaly of the flexures“) handelt es sich um eine seltene Genodermatose mit autosomal-dominantem Erbgang von unterschiedlicher Penetranz, der eine Störung der epidermalen Proliferation zugrunde liegt. Klinisch ist der „klassische“ Morbus Dowling-Degos durch eine netzartige Pigmentierung inguinal und axillär, später auch anderer Beugen des Körpers gekennzeichnet. Bei der hier vorgestellten Patientin sowie auch bei ihrer Mutter liegt eine ausschließlich genitoperianale Manifestation vor.

\section{Kasuistik}

\section{Patientin 1 (Tochter)}

Eine 31-jährige Patientin wurde uns von ihrem behandelnden Gynäkologen zur Abklärung von Hyperpigmentierungen genital und perianal überwiesen. Über den Zeitpunkt der Entstehung dieser Hyperpigmentierungen konnte die Patientin keine ge- naueren Angaben machen. Sie wusste auch nicht, ob ähnliche Hautveränderungen bei weiteren Familienmitgliedern bestehen.

Befund: Im Bereich der großen und kleinen Labien sowie perianal bestehen braune, gering über Hautniveau erhabene Hyperpigmentierungen, die durch Konfluenz ein netzartiges Muster bilden (Abb. 1 au.b). Am restlichen Integument besteht ein unauffälliger Hautbefund; die internistisch-orientierende Untersuchung zeigt keine Auffälligkeiten.

Histopathologie: Biopsie von der großen Labie: Die Epidermis zeigt eine Orthohyperkeratose, Akanthose mit schmalzapfig verlängerten Reteleisten. Die epidermalen Zellen sind basal deutlich hyperpigmentiert. Vereinzelt finden sich Pseudohornzysten (Abb. 2).

Immunhistochemie: Die Färbung mit Antikörpern gegen Melan-A zeigt keine Vermehrung von Melanozyten (Abb.3).

Diagnose: Morbus Dowling-Degos in ausschließlich genitoperianaler Lokalisation. 


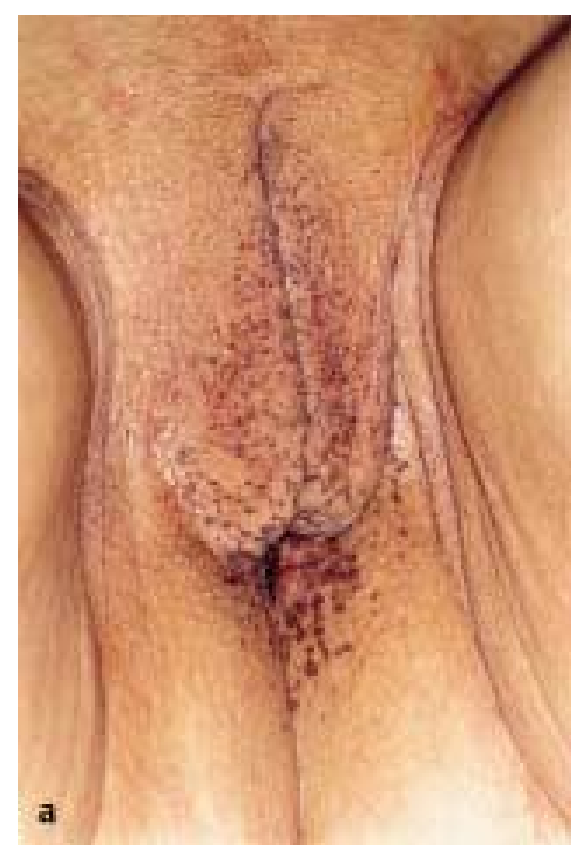

Abb. $\mathbf{1}$ a, b Retikuläre Hyperpigmentierungen genital (Patientin1).

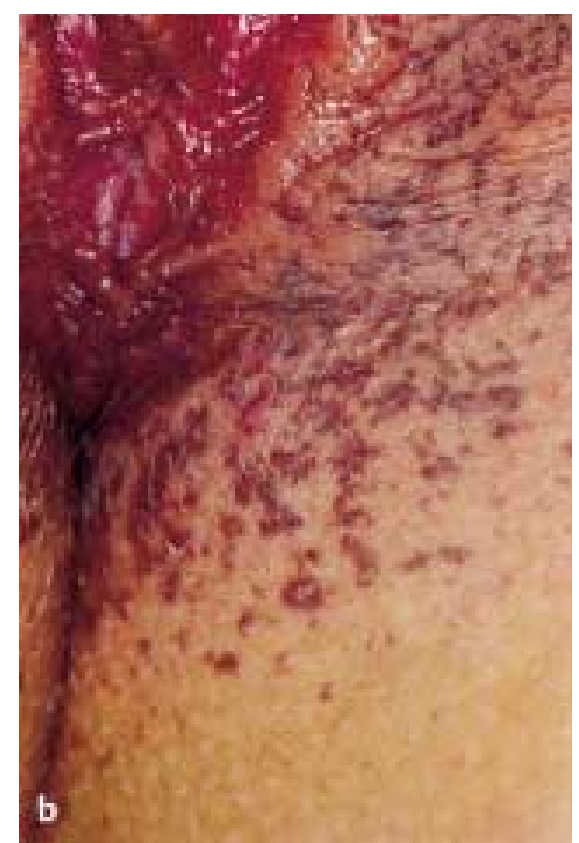

\section{Patientin 2 (Mutter)}

Die 50-jährige Mutter von Patientin 1 stellte sich zur Kontrolle der bei ihr seit dem 20. Lebensjahr bekannten Neurofibromatosis von Recklinghausen vor.

Befund: Bei der Ganzkörperinspektion zeigen sich neben den Veränderungen im Rahmen der bekannten Neurofibromatose (multiple Neurofibrome am gesamten Integument, Café au laitFlecken und „axillary freckling“) ähnlich wie bei ihrer Tochter retikuläre Hyperpigmentierungen im Bereich der Labien und perianal (Abb. 4).

Diagnosen: Morbus Dowling-Degos in ausschließlich genitoperianaler Lokalisation sowie Neurofibromatosis Recklinghausen.

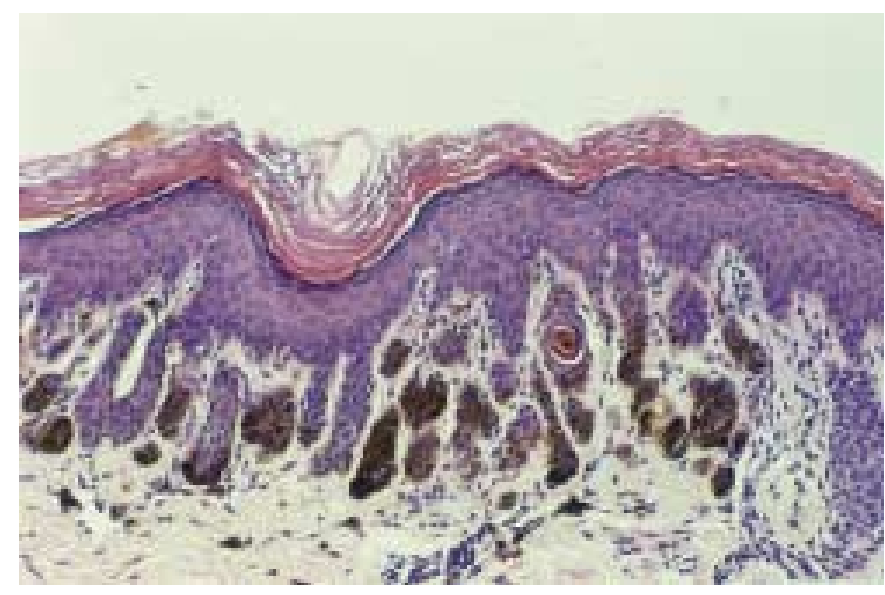

Abb. 2 Histologie (Patientin1): Akanthose der Epidermis mit ausgezogenen hyperpigmentierten Reteleisten, Hyperkeratose und Pseudohornzyste.

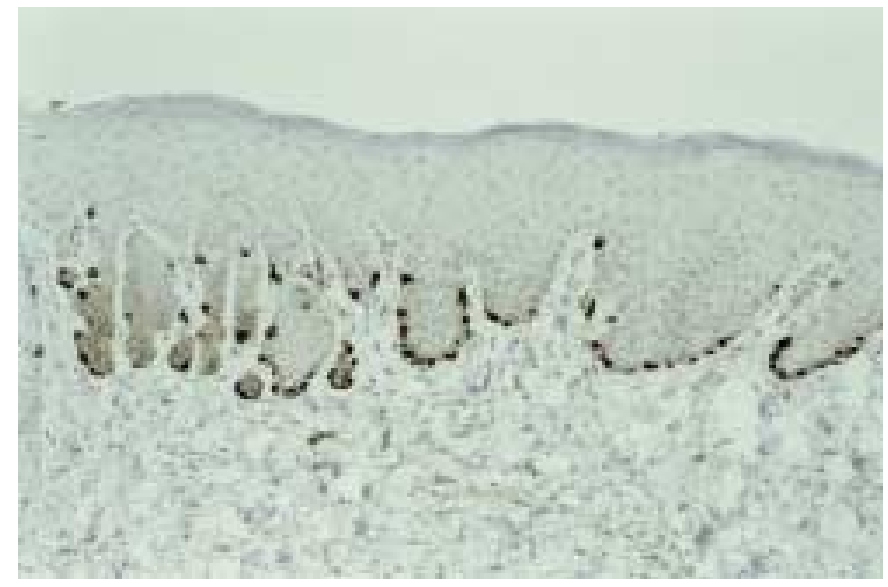

Abb. 3 Immunhistologie: keine Vermehrungvon Melanozyten (Melan A).

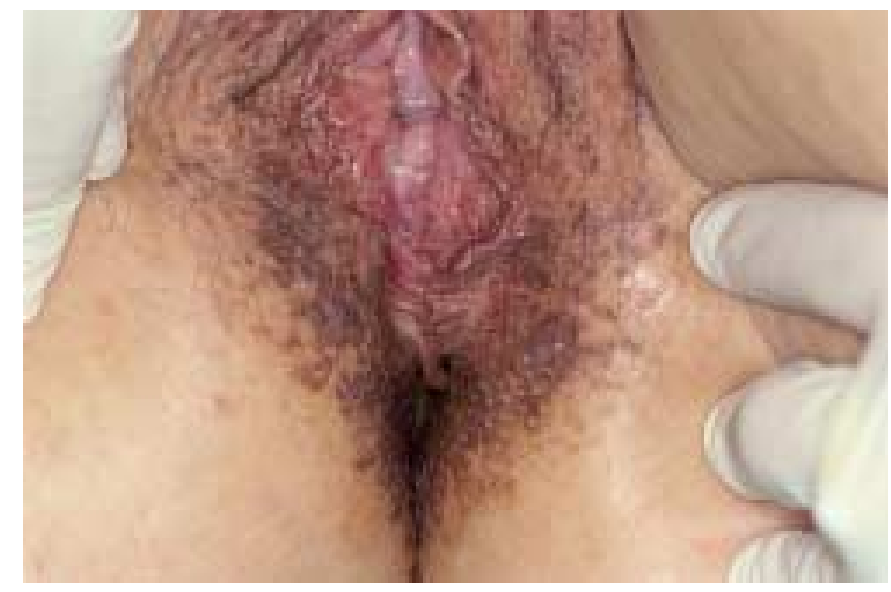

Abb. 4 Retikuläre Hyperpigmentierungen genital (Patientin 2). 
Besprechung

Wilson-Jones und Grice prägten 1978 den Namen „Morbus Dowling-Degos („reticulate pigmented anormaly of the flexures“), der auf Dowling und Freudenthal zurückgeht, die die Erkrankung 1938 erstmals als klinische Entität abgrenzten und auf Degos und Ossipowski, die sie 1954 unter der Bezeichnung „Dermatose pigmentaire reticulée des plis“ erneut beschrieben [1,2]. Die Erkrankung stellt eine seltene Genodermatose mit autosomal-dominantem Erbgang von unterschiedlicher Penetranz dar [3,4]. Die typische Klinik besteht aus gesprenkelten bis netzförmigen Hyperpigmentierungen der Haut vor allem in den großen Gelenkbeugen und den Intertrigines. Dowling beschreibt in dem 1938 von ihm veröffentlichten Fall, dass die Pigmentierungen genital dunkler und nahezu konfluierend, axillär jedoch schwächer ausgeprägt waren. Unsere Patientinnen weisen ausschließlich Pigmentierungen im Vulva- und Perianalbereich auf. Ähnliche Fälle sind vereinzelt in der Literatur beschrieben [5].

Histopathologisch typisch für den Morbus Dowling-Degos sind eine Akanthose der Epidermis mit ausgezogenen hyperpigmentierten Reteleisten, Hyperkeratose und pseudozystische Horneinschlüsse.

Einige Autoren ordnen den Morbus Dowling-Degos in den Formenkreis der „umschriebenen retikulären Hyperpigmentierungen“ ein, zu denen sie noch weitere in der Literatur ursprünglich eigenständig beschriebene Erkrankungen zählen. Neben der allen Erkrankungen gemeinsamen netzartigen Hyperpigmentierung zeigen sich weitere epitheliale Veränderungen unterschiedlicher Ausprägung. Dabei findet sich am einen Ende des Spektrums dieser Erkrankungen der Morbus Kitamura, der klinisch lediglich retikuläre Hyperpigmentierungen aufweist. Das andere Ende des Spektrums bilden die Pigmentatio reticularis faciei et colli mit epithelialer Zystomatose und die familiär auftretenden multiplen follikulären Hamartome. Zwischen diesen beiden Endpunkten werden das Haber-Syndrom, der Morbus Dowling-Degos und der Morbus Galli-Galli - eine akantholytische Variante des Morbus Dowling-Degos - gezählt (Tab.1) [6,7].

Tab. 1 Umschriebene retikuläre Hyperpigmentierungen nach [6]

- Morbus Kitamura

- Morbus Dowling-Degos

- Morbus Galli-Galli

- Haber-Syndrom

- Pigmentatio reticularis faciei et colli mit epithelialer Zystomatose

- familiär auftretende multiple follikuläre Hamartome
Differenzialdiagnostisch muss beim „klassischen“ Morbus Dowling-Degos die Acanthosis nigricans, aufgrund des familiären Vorkommens insbesondere die Acanthosis nigricans benigna familiaris, in Erwägung gezogen werden; wobei die Acanthosis nigricans klinisch meist aufgrund des kopfsteinpflasterartigen Oberflächenreliefs sowie des fehlenden retikulären Musters unterschieden werden kann. Des Weiteren sind die Papillomatosis confluens et reticularis (Gougerot und Carteau) [8] und die sommersprossenartigen Hyperpigmentierungen (,axillary freckling“) bei Neurofibromatosis von Recklinghausen (die bei Patientin 2 im Rahmen des bei ihr bekannten Morbus Recklinghausen zusätzlich bestand) in die differenzialdiagnostischen Überlegungen miteinzubeziehen. Bei Befall des Genitoanalbereiches sollte differenzialdiagnostisch insbesondere an die Melanosis der Vulva [9] und die Lentiginosis profusa perigenitoaxillaris [10] gedacht werden. Histopathologisch kann insbesondere die Abgrenzung zur flachen bzw. adenoiden seborrhoischen Keratose schwierig sein.

Bisher besteht kein erfolgreiches therapeutisches Konzept in der Behandlung des asymptomatischen, lediglich kosmetisch störenden Morbus Dowling-Degos. Die lokale und systemische Anwendung von Retinoiden führte nicht zu einer verifizierbaren Besserung der Dermatose [11]. Es wurde vereinzelt über gute kosmetische Ergebnisse nach einer Laserbehandlung berichtet. Bei den beiden hier vorgestellten Patientinnen bestand kein Leidensdruck und folglich auch kein Therapiewunsch.

\section{Literatur}

${ }^{1}$ Wilson-Jones E, Grice K. Reticulate pigmented anormaly of the flexures. Dowling Degos disease, a new genodermatosis. Arch Dermatol 1978; 114: 1150 - 1157

${ }^{2}$ Degos R, Ossipowski B. Dermatose pigmentaire reticulée des plis. Ann Dermatol Syphilol 1954; 81: 147 - 151

${ }^{3}$ Crovato F, Nazzari N, Rebora A. Dowling Degos disease (reticulate pigmented anormaly of the flexures) is an autosomal dominant condition. Br J Dermatol 1983; 108: 473-476

${ }^{4}$ Blitz H, Kießling M. Morbus Dowling-Degos - eine autosomal dominante Genodermatose. Z Hautkr 1988; 63: 642 - 644

${ }^{5}$ Milde P, Goerz G, Plewig G. Morbus Dowling-Degos mit ausschließlich genitaler Manifestation. Hautarzt 1992; 43: 369-372

${ }^{6}$ Schiller M, Kütting L, Metze D. Umschriebene retikuläre Hyperpigmentierungen - ein weites klinisches Spektrum. Hautarzt 1999; 50: $580-585$

${ }^{7}$ Rebora A, Crovota F. The spectrum of Dowling Degos disease. Brit J Dermatol 1984; 110: 627-630

${ }^{8}$ Gougerot H, Carteaud A. Papillomatose pigmentee innominee. Bull Soc Fr Dermatol Syphiligr 1927; 34: 719-721

9 Jackson R. Melanosis of the vulva. J Dermatol Surg Oncol 1984; 10: $119-121$

${ }^{10}$ Korting GW. Lentiginosis profusa perigenito-axillaris. Z Hautkr 1967; 42: XIX-XXII

11 Oppolzer G, Schwarz T, Duschet P, Brenner W, Gschnait FM. DowlingDegos: frustraner Therapieversuch mit Retinoiden. Hautarzt 1987; 38 : $615-618$ 